\title{
Tratamento com Antiinflamatórios Tópicos na Osteoartrite de Joelho
}

\section{Topical Anti-inflammatory Drugs in Osteoarthritis of the Knee}

\author{
Helena Lúcia Alves Pereira ${ }^{(1)}$, Sandra Lúcia Euzébio Ribeiro ${ }^{(2)}$, Rozana Mesquita Ciconelli $^{(3)}$
}

\section{RESUMO}

Os antiinflamatórios não-esteroidais (AINEs) orais são eficazes no alívio da dor na osteoartrite (OA), porém têm o risco de efeitos adversos sistêmicos. Para diminuir a toxicidade é necessário reduzir a concentração plasmática uma vez que os efeitos adversos são dose-dependentes. Os AINEs de uso tópico, em teoria, alcançam altas concentrações nos tecidos-alvo com concentrações plasmáticas baixas, sendo portanto uma alternativa terapêutica, especialmente para pacientes de risco. Nossa revisão dos ensaios clínicos randomizados mostrou que os AINEs tópicos foram mais efetivos que o placebo e tão eficazes quanto os AINEs orais. Porém, o pequeno número de ensaios e falhas na metodologia nos impossibilita de fazer conclusões definitivas, sendo necessários estudos bem desenhados e de maior duração.

Palavras-chave: AINEs tópicos, osteoartrite, ensaios clínicos.

\section{INTRODUÇÃO}

A osteoartrite (OA) é a doença articular mais prevalente e também a principal causa de dor e incapacidade na população idosa ${ }^{(1)}$. O tratamento deve ser multidisciplinar e a orientação para o controle da OA de joelho tem sido publicada em diversos países, inclusive no Brasil $^{(2-4)}$.

Medidas não-farmacológicas incluem programas educacionais, exercícios terapêuticos, órteses e equipamentos de auxílio à marcha. $\mathrm{O}$ tratamento farmacológico deve ser multifatorial, uma vez que a prescrição de um agente terapêutico isolado pode não ser suficiente para o controle da dor. Os analgésicos, como o paracetamol, são considerados agentes de primeira escolha. Antiinflamatórios são indicados nos casos de processo inflamatório agudo. Analgésicos opióides, terapia intra-articular com glicocorticóides e derivados do ácido hialurônico também estão indicados. Apesar da recomendação, os antiinflamatórios não-esteroidais (AINEs) orais continuam a ser largamente utilizados

\begin{abstract}
Oral non-steroidal anti-inflammatories drugs (NSAIDs) are effective in pain relief in osteoarthritis (OA), but are also associated with risks of adverse systemic side effects. In order to reduce its toxicity, it would be desirable to decrease the plasmatic levels of NSAIDs since those side effects are dose-dependent. Topical NSAIDs would be a therapeutic alternative in theory, because while they reach high levels in local tissues, they also produce low plasmatic levels. Our review analyzed clinical randomized and shown that topical NSAIDs were superior to placebo and have similar efficacy to oral NSAIDs, but due a few number of trials and methodological weaknesses we can't make definitive conclusions. Further well designed, long term studies are required.
\end{abstract}

Keywords: topical NSAIDs, osteoarthritis, clinical trials.

com ou sem prescrição médica. Porém, seu uso tem o risco da toxicidade, com 2 a $4 \%$ de incidência anual de sérias complicações gastrintestinais, quatro vezes maior do que nos indivíduos que não usam ${ }^{(5)}$. Como os efeitos adversos são dose-dependente, a redução dos níveis plasmáticos seria desejável, desde que se mantivesse o efeito terapêutico. A aplicação tópica de AINEs com alta penetração percutânea ofereceria altas concentrações nos tecidos-alvo com menor índice de efeitos colaterais sistêmicos. A cartilagem articular é desprovida de inervação. Portanto, a dor provavelmente se origina de tecidos periarticulares e da cápsula articular, apesar do mecanismo exato não estar esclarecido.

Os AINEs têm sido usados topicamente por décadas para aliviar a dor em tecidos musculoesqueléticos ${ }^{(6)}$. Esta via de administração possivelmente reduz as reações adversas por maximizar o efeito local e minimizar a toxicidade sistêmica. O principal problema tem sido a questão da penetração no tecido-alvo e, portanto, a eficácia clínica.

\footnotetext{
Recebido em 23/05/05. Aprovado, após revisão, em 26/01/06.

1. Médica Reumatologista Assistencial do Hospital Universitário Getúlio Vargas da Universidade Federal do Amazonas (UFAM) e pós-graduanda da Disciplina de Reumatologia da Universidade Federal de São Paulo (UNIFESP).

2. Professora do Departamento da Clínica Médica da Disciplina de Reumatologia da UFAM e pós-graduanda da Disciplina de Reumatologia da UNIFESP.

3. Médica Assistente Doutora da Disciplina de Reumatologia do Departamento de Medicina da UNIFESP

Endereço para correspondência: Helena Lucia Alves Pereira, Rua Apurinã, 04, Praça 14 de Janeiro, CEP 69020-170, Manaus, AM, Brasil, e-mail: hlucia@vivax.com.br
} 
Para se obter eficácia, postula-se que o acúmulo local da droga nos tecidos-alvo, que ocorre por difusão direta, deva ter concentrações maiores do que a obtida por absorção sistêmica e redistribuição através da vasculatura cutânea. A concentração plasmática máxima obtida após aplicação tópica é menor que $15 \%$ da concentração obtida após administração oral.

Estudos em animais confirmam esta hipótese. McNeill et $\mathrm{al}^{7)}$ demonstraram que após aplicação tópica e intravenosa de piroxicam, no músculo subjacente à aplicação tópica, foram detectados dois picos de concentração 4 e 12 horas após a aplicação, enquanto que, no músculo contralateral, houve apenas um pico de 12 horas, sugerindo que o primeiro pico foi produzido pela absorção tópica e o segundo, pela redistribuição sistêmica. A concentração do antiinflamatório obtida no músculo subjacente à aplicação tópica foi 14 vezes maior que a do músculo contralateral. Mikulak et a ${ }^{(8)}$ relataram que a aplicação tópica de indometacina produziu uma concentração máxima no músculo subjacente e cápsula articular de dois a dez vezes maior que a concentração obtida pela administração oral.

A absorção transcutânea depende da natureza da substância, do veículo e das condições da pele. Há uma grande variabilidade individual na absorção, atribuída à hidratação da pele, características de permeabilidade e diferenças individuais na vasculatura subcutânea ${ }^{(9)}$. A substância considerada ideal deve ter baixo peso molecular, potência elevada e propriedades hidrofilicas para penetração no estrato córneo, lipofilico e hidrofóbicas para penetrar camada epidérmica, aquosa ${ }^{(10)}$. O tipo de formulação também é importante. As propriedades do veículo podem aumentar a absorção até cinco vezes.

\section{ENSAIOS CLÍNICOS COM AINES TÓPICOS}

Os ensaios clínicos sobre a eficácia dos AINEs tópicos no alívio da dor na OA de joelhos são de difícil interpretação por falta de estudos relevantes. Os estudos existentes são de validade questionável por apresentarem falhas metodológicas quanto ao desenho do estudo, tamanho da amostra, documentação da aderência ao tratamento, técnica de aplicação, formulação das preparações, doses inadequadas. Outras questões a serem mencionadas são o uso de analgesia de resgate e a alta resposta ao placebo. O rubor produzido na região dolorosa após aplicação tópica tem efeitos benéficos na dor. Esta revisão foi realizada a partir de uma busca da literatura no período de 1980 a 2005, através do uso da base de dados Medline. Foram incluídos somente estudos randomizados controlados, uma revisão sistemática e uma metanálise.

\section{ENSAIOS COM ANTIINFLAMATÓRIOS TÓPICOS VERSUS PLACEBO}

Nossa revisão identificou 11 ensaios potencialmente relevantes em OA de joelho. A Tabela 1 mostra a metodologia e os resultados dos estudos. Algozzine et al ${ }^{(1)} \mathrm{e}$ Shackel et a ${ }^{(12)}$ compararam salicilato de trolamina e salicilato de cobre versus placebo. Em ambos os estudos não encontraram diferença significativa nas medidas objetivas e subjetivas de alívio da dor entre os grupos.

Dreiser et $\mathrm{al}^{(13)}$, Grace et a ${ }^{(14)}$ e Bruhlmann et $\mathrm{al}^{(15)} \mathrm{com}$ pararam diclofenaco na forma de emplastro ou gel versus placebo. Nos estudos, ambos os grupos (droga e placebo) melhoraram, porém os melhores resultados foram obtidos com o grupo do diclofenaco, com diferença estatística. No estudo de Ottilinger et al ${ }^{(16)}$, foi utilizado eltenaco gel em várias concentrações comparado ao placebo. $\mathrm{O}$ autor não encontrou diferença estatística entre os grupos na análise das medidas de desfecho primário (dor pela escala visual analógica e índice de severidade de Lequesne $)^{(17)}$. Embora tenha sido detectada uma tendência para a superioridade do eltenaco na maior concentração, esta não alcançou diferença estatística. Ibuprofeno creme a $5 \%$ foi a droga testada contra placebo tópico nos estudos de Rovensky ${ }^{(18)} \mathrm{e} \mathrm{Trnavsky}^{(19)} \mathrm{e}$ os resultados foram favoráveis ao ibuprofeno.

Bookman et $a^{(20)}$, Roth e Shainhouse ${ }^{(21)}$ e Baer et al ${ }^{(22)}$ realizaram ensaios clínicos nos quais testaram solução de diclofenaco comparado ao placebo. Foi utilizado como veículo DMSO (dimetil sulfóxido), substância que in vitro aumenta a penetração percutânea do antiinflamatório. Os estudos adotaram os parâmetros de avaliação preconizados pelo OMERACT III (Outcome Measures in Rheumatoid Arthritis Clinical Trials) e OARSI (Osteoarthritis Research Society International) ${ }^{(23)}$. Ambos os ensaios demonstraram superioridade do diclofenaco nas medidas de avaliação primária (alívio da dor medido pelo WOMAC) ${ }^{(24)}$ e secundária. A incidência de efeitos colaterais locais foi maior no grupo diclofenaco, porém os efeitos sistêmicos foram de baixa incidência e semelhante nos grupos.

Conforme mostrado na Tabela 1 , nos ensaios em que a duração foi de no máximo duas semanas, os resultados se mostraram favoráveis aos AINEs tópicos. Nos ensaios com maior duração ( 4 a 12 semanas), três foram favoráveis à droga e em outros dois os resultados não demonstraram diferença estatística. Na maioria dos estudos, a droga e o placebo foram disponibilizados por laboratórios farmacêuticos e, nos três últimos ensaios, um dos participantes estava ligado à indústria farmacêutica. 
TABELA 1

CARACTERÍSTICAS DOS ENSAIOS CLÍNICOS RANDOMIZADOS CONTROLADOS COMPARANDO ANTIINFLAMATÓRIOS NÃO-ESTERÓIDES (AINEs) TÓPICOS COM PLACEBO, EM PACIENTES COM OSTEOARTRITE (OA) DE JOELHO

\begin{tabular}{|c|c|c|c|c|c|c|}
\hline Ensaio & Desenho & Sítio da OA & $\begin{array}{l}\text { Duração } \\
\text { (semanas) }\end{array}$ & $\begin{array}{l}\text { AINE Tópico } \\
\text { (n pacientes) }\end{array}$ & Controle (n pacientes) & Conclusão \\
\hline Algozzine, 1982 & $\begin{array}{l}\text { Duplo-cego } \\
\text { crossover }\end{array}$ & Joelho & 1,1 , e 1 & $\begin{array}{l}\text { Salicilato } \\
4 \times \text { dia }(26)\end{array}$ & Placebo (26) & $\begin{array}{c}\text { Salicilato igual ao } \\
\text { placebo }\end{array}$ \\
\hline Dreiser, 1993 & $\begin{array}{l}\text { Duplo-cego } \\
\text { paralelo }\end{array}$ & Joelho & 2 & $\begin{array}{l}\text { Diclofenaco } \\
3 \times \text { dia }(78)\end{array}$ & Placebo (77) & $\begin{array}{l}\text { Diclofenaco melhor } \\
\text { que placebo }\end{array}$ \\
\hline Shackel, 1997 & $\begin{array}{l}\text { Duplo-cego } \\
\text { paralelo }\end{array}$ & $\begin{array}{l}\text { Joelho/ } \\
\text { quadril }\end{array}$ & 4 & Salicilato 2x dia (58) & Placebo (58) & $\begin{array}{c}\text { Salicilato igual ao } \\
\text { placebo }\end{array}$ \\
\hline Grace, 1999 & $\begin{array}{l}\text { Duplo-cego } \\
\text { paralelo }\end{array}$ & Joelho & 2 & Diclofenaco (38) & Placebo (36) & $\begin{array}{l}\text { Diclofenaco melhor } \\
\text { que placebo }\end{array}$ \\
\hline Ottillinger, 2001 & $\begin{array}{l}\text { Duplo-cego } \\
\text { paralelo }\end{array}$ & Joelho & 4 & $\begin{array}{c}\text { Eltenaco: } \\
3 \mathrm{mg} 3 \times \text { dia (59), } \\
9 \mathrm{mg} 3 \times \text { dia (59), } \\
30 \mathrm{mg} 3 \times \text { dia (57) }\end{array}$ & $\begin{array}{c}\text { Placebo (59), placebo (59), } \\
\text { placebo (59) }\end{array}$ & $\begin{array}{l}\text { Eltenaco igual a } \\
\text { placebo }\end{array}$ \\
\hline Bruhlmann, 2003 & $\begin{array}{l}\text { Duplo-cego } \\
\text { paralelo }\end{array}$ & Joelho & 2 & $\begin{array}{l}\text { Diclofenaco } \\
2 \times \text { dia }(51)\end{array}$ & Placebo (52) & $\begin{array}{l}\text { Diclofenaco melhor } \\
\text { que placebo }\end{array}$ \\
\hline Rovensky, 2003 & $\begin{array}{l}\text { Duplo-cego } \\
\text { paralelo }\end{array}$ & Joelho & 1 & $\begin{array}{l}\text { Ibuprofeno 5\% } \\
200 \mathrm{mg} 3 \times \text { dia }\end{array}$ & Placebo $3 x$ dia & $\begin{array}{l}\text { Ibuprofeno melhor } \\
\text { que placebo }\end{array}$ \\
\hline Trnavsky, 2004 & $\begin{array}{l}\text { Duplo-cego } \\
\text { Paralelo }\end{array}$ & Joelho & 1 & $\begin{array}{l}\text { Ibuprofeno } 5 \% \\
200 \mathrm{mg} 3 \times(25)\end{array}$ & Placebo $3 x$ dia (25) & $\begin{array}{l}\text { Ibuprofeno melhor } \\
\text { que placebo }\end{array}$ \\
\hline Roth, 2004 & Duplo-cego & Joelho & 12 & $\begin{array}{l}\text { Solução de diclofenaco } \\
\text { + DMSO } 4 \text { x dia (119) }\end{array}$ & $\begin{array}{l}\text { Solução placebo + DMSO } \\
\text { (109) }\end{array}$ & $\begin{array}{l}\text { Diclofenaco melhor } \\
\text { que placebo }\end{array}$ \\
\hline Bookman, 2004 & Duplo-cego & Joelho & 4 & $\begin{array}{l}\text { Solução de diclofenaco } \\
\text { + DMSO } 4 \times \text { dia (82) }\end{array}$ & placebo + DMSO 45,5\% (82) & Diclofenaco melhor \\
\hline Baer, 2005 & Duplo-cego & Joelho & 4 & $\begin{array}{l}\text { Solução de diclofenaco } \\
+ \text { DMSO } 4 \mathrm{x} \text { dia }\end{array}$ & Placebo + DMSO 4 x dia & $\begin{array}{l}\text { Diclofenaco melhor } \\
\text { que placebo }\end{array}$ \\
\hline
\end{tabular}

\section{REVISÃO SISTEMÁTICA / METANÁLISE}

Moore et $a^{(25)}$ realizaram uma revisão quantitativa dos ensaios clínicos avaliando a efetividade e segurança dos AINEs tópicos para tratamento de uma variedade de condições agudas e crônicas, incluindo OA.

Foram selecionados 12 estudos em condições crônicas (OA/tendinite) comparando AINEs tópicos versus placebo, com 547 pacientes em tratamento com droga e $550 \mathrm{em}$ tratamento com placebo. Os autores incluíram somente estudos controlados randomizados, com duração de duas semanas, nos quais a redução da dor era o principal desfecho. Uma significativa melhora do tratamento em comparação ao controle foi considerada quando o benefício relativo foi maior que 1 .

Os principais achados foram: 1) em oito estudos, os AINEs tópicos foram significativamente melhores que placebo em duas semanas de tratamento, com um risco relativo (RR) para o benefício de 2,0 (IC 95\% - 1,5 a 2,7) e um número necessário para tratar (NNT) de 3,1 (2,7 a $3,8)$, ou seja, para cada três pacientes tratados um obteve benefício; 2) os efeitos adversos e abandono de tratamento com os AINEs tópicos foram de baixa incidência, não havendo diferença entre o tratamento e o placebo.

A Figura 1 mostra o benefício relativo para cada ensaio controlado e a combinação de todos. O diamante representa o benefício relativo dos dados combinados e as barras representam o intervalo de confiança de $95 \%$. Todas as comparações ficaram no segmento favorecendo o tratamento.

Lin et al ${ }^{(26)}$ realizaram uma metanálise sobre a eficácia dos AINEs tópicos somente na OA. Das 133 citações encontradas na literatura, somente 13 estudos representando 1983 pacientes preencheram os critérios de inclusão, sendo então selecionados para análise. Todos os estudos eram controlados randomizados comparando 
AINEs tópicos com placebo ou AINEs orais. A taxa de abandono foi de 1 a $23 \%$.

O desfecho primário foi redução da dor (dor global ou dor em repouso). Outras medidas de desfecho foram a função e a rigidez. Os principais resultados foram: 1) AINEs tópicos foram superiores ao placebo no alívio da dor nas duas primeiras semanas de tratamento, porém nas duas semanas seguintes não houve diferença estatística entre o grupo tratado e o placebo; 2) o mesmo foi observado quanto a função e rigidez articular; 3 ) AINEs tópicos foram menos efetivos que o oral numericamente em qualquer semana e estatisticamente na primeira semana; 4) quanto aos efeitos adversos, o tratamento foi equivalente ao placebo. $\mathrm{O}$ autor admite que houve muita heterogeneidade nos estudos, que poderia contribuir para um viés, em favor do tratamento sobre o placebo. O estudo também detectou que a classe dos AINEs também influenciou o resultado, sendo que ibuprofeno, diclofenaco, eltenaco e ácido salicílico obtiveram melhores resultados.

A Figura 2 mostra o resultado de cada ensaio e a combinação de todos. Observa-se que em quatro estudos os AINEs tópicos foram significativamente melhores que o placebo e, na combinação de todos, os resultados obtidos foram favoráveis aos AINEs tópicos nas duas semanas iniciais de tratamento. Porém, nos ensaios que se estenderam até quatro semanas, a efetividade do tratamento foi igual ao placebo.

Figura 1

REVISÃo SISTEMÁticA DOS AINES TÓPICOS(25)

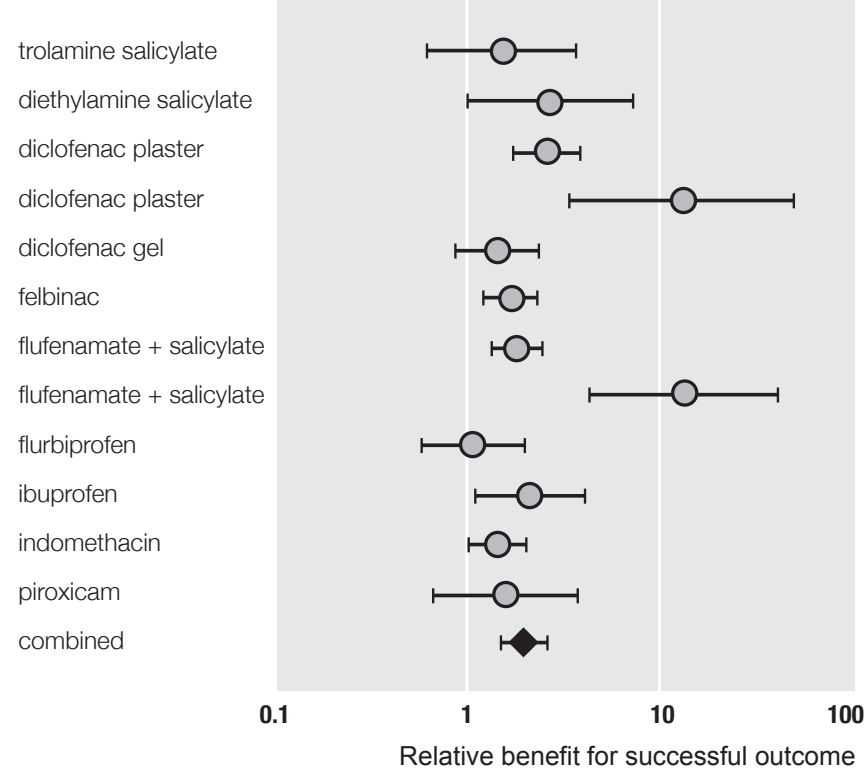

Figura 2

AINES TÓPICOS NO TRATAMENTO DA OSTEOARTRITE: METANÁLISE DOS ESTUdOS CONTROLADOS E RANDOMIZADOS ${ }^{(26)}$
Week 1

Brulmann $2003^{35}$ Dreiser $1993^{18}$ Ottillinger $2001^{37}$ Ottillinger $2001^{37}$ Roth $1995^{38}$

Rothacker $1998^{22}$ Rovensky $2001^{23}$ Sandelin $1997^{24}$ Pooled

Fixed effect Random effect

Week 2

Brulmann $2003^{35}$ Dreiser $1993^{18}$ Grace $1999^{20}$ Ottillinger $2001^{37}$ Ottillinger $2001^{37}$ Roth $1995^{3 \mathrm{~B}}$

Sandelin $1997^{24}$ Pooled

Fixed effect Random effect

Week 3

Ottillinger $2001^{37}$ Ottillinger $2001^{37}$ Ottillinger $2001^{37}$ Sandelin $1997^{24}$ Pooled

Fixed effect Random effect

\section{Week 4} Ottillinger $2001^{37}$ Sandelin $1997^{24}$ Shackel $1997^{40}$ Pooled Fixed effect Random effect

$-1$ Favours placebo Ottillinger $2001^{37}$ Ottillinger $2001^{37}$

Ottillinger $2001^{37}$ Ottillinger $2001^{37}$
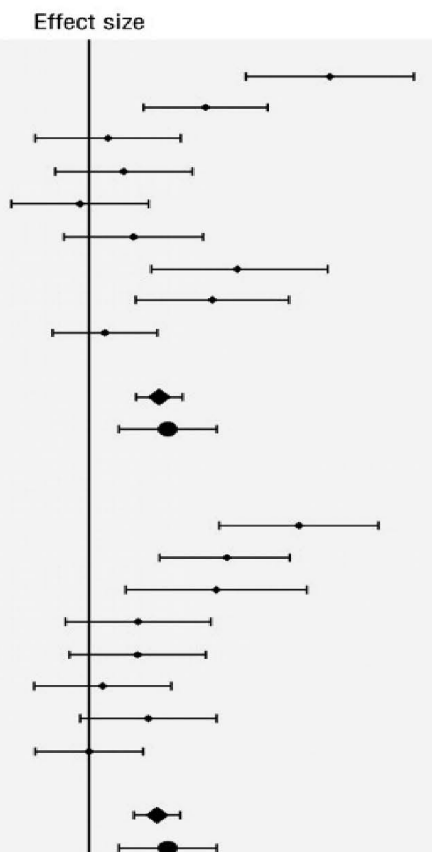

\section{ENSAIOS CLÍNICOS COMPARANDO AINES TÓPICOS VERSUS AINES ORAIS}

Há poucos estudos comparando os AINEs tópicos com os orais na OA. A Tabela 2 apresenta o resultado de quatro estudos nos quais a preparação tópica foi tão efetiva quanto a oral. Nos ensaios de Tsuyama et al ${ }^{(27)}$, Sandelin et $\mathrm{a}^{(28)}$ e Dickson ${ }^{(29)}$, os pacientes obtiveram uma melhora 
TABELA 2

CARACTERÍSTICAS dOS ENSAIOS CLÍNICOS RANDOMIZADOS CONTROLADOS COMPARANDO AINES TÓPICOS COM AINES ORAIS EM PACIENTES COM OSTEOARTRITE (OA)

\begin{tabular}{|c|c|c|c|c|c|c|c|}
\hline Ensaio & Desenho & Sítio da OA & $\begin{array}{l}\text { Duração } \\
\text { (semanas) }\end{array}$ & Tratan & nento & $\begin{array}{c}\text { Total } \\
\text { (n pacientes) }\end{array}$ & Conclusão \\
\hline Tsuyama, 1985 & $\begin{array}{l}\text { Duplo-cego } \\
\text { paralelo }\end{array}$ & Joelho & 2 & $\begin{array}{l}\text { Felbinaco Tópico + } \\
\text { Placebo oral } 3 \times \text { dia }\end{array}$ & $\begin{array}{c}\text { Fenbufen oral } 200 \\
\mathrm{mg}+\text { Gel Placebo } \\
3 \times \text { dia }\end{array}$ & 275 & $\begin{array}{c}\text { Sem diferença } \\
\text { estatística }\end{array}$ \\
\hline Sandelin, 1997 & $\begin{array}{l}\text { Duplo-cego } \\
\text { paralelo }\end{array}$ & Joelho & 4 & $\begin{array}{c}\text { Eltenaco Tópico } \\
\text { 30mg + Placebo oral } \\
2 \times \text { dia }\end{array}$ & $\begin{array}{c}\text { Diclofenaco oral } 50 \\
\text { mg } 2 \times \text { dia }+ \text { Gel } \\
\text { Placebo } 3 \times \text { dia }\end{array}$ & 208 & $\begin{array}{c}\text { Sem diferença } \\
\text { estatística }\end{array}$ \\
\hline Dickson, 1991 & $\begin{array}{l}\text { Duplo-cego } \\
\text { paralelo }\end{array}$ & Joelho & 4 & $\begin{array}{l}\text { Piroxicam Tópico } \\
5 \mathrm{mg} 3 \times \text { dia }\end{array}$ & $\begin{array}{l}\text { Ibuprofeno oral } 400 \\
\text { mg } 3 \times \text { dia }\end{array}$ & 235 & $\begin{array}{c}\text { Sem diferença } \\
\text { estatística }\end{array}$ \\
\hline Tugwell, 2004 & $\begin{array}{l}\text { Duplo-cego } \\
\text { paralelo }\end{array}$ & Joelho & 12 & $\begin{array}{l}\text { Diclofenaco Tópico + } \\
\text { Placebo oral } 3 \times \text { dia }\end{array}$ & $\begin{array}{l}\text { Placebo tópico + } \\
\text { Diclofenaco oral } \\
50 \mathrm{mg} 3 \times \text { dia }\end{array}$ & 622 & $\begin{array}{c}\text { Sem diferença } \\
\text { estatística }\end{array}$ \\
\hline
\end{tabular}

na dor e na capacidade funcional ao final dos experimentos em ambos os grupos não havendo diferença estatística. Entretanto, a metodologia foi inadequada. A dose do AINE oral foi abaixo da dose preconizada, a duração do estudo foi de no máximo quatro semanas e compararam AINEs de diferentes classes.

Tugwell et $a^{(30)} \mathrm{em}$ estudo recente, com duração de 12 semanas, compararam solução de diclofenaco tópico com diclofenaco oral, demonstraram que a solução tópica e a oral obtiveram melhora equivalente na dor, capacidade funcional e avaliação global do paciente $(36 / 44 \%$ versus $42 / 49 \%$ ). Apesar de uma pequena diferença a favor do diclofenaco oral, esta não foi significativa. Dos pacientes com efeitos adversos sistêmicos, $48 \%$ foram do grupo diclofenaco oral e $35 \%$ do grupo diclofenaco tópico. No total, $28 \%$ dos pacientes do grupo oral e $30 \%$ do grupo tópico foram retirados do estudo por efeitos adversos e ineficácia. Este estudo é importante porque comparou a mesma droga, incluiu grande número de pacientes, aderiu aos princípios recomendados para um estudo de equivalência e foi desenhado segundo as recomendações da OARSI para ensaios clínicos em OA (maior duração, critérios de inclusão clínicos e radiológicos bem definidos e parâmetros de avaliação e desfecho clínicos preconizados pelo OMERACT).

\section{CONSIDERAÇÕES FINAIS}

A toxicidade dos AINEs orais é um problema sério, principalmente na população idosa, a mais acometida pela OA. Apesar do uso de inibidores seletivos da COX-2 reduzirem o risco de efeitos adversos gastrintestinais, o perfil de segurança dessas drogas atualmente está sendo questionado devido a outros efeitos adversos graves, como o aumento da incidência de eventos cardiovasculares trombóticos ${ }^{(31)}$.

Os AINEs tópicos, ao agirem diretamente no local da aplicação, mantendo níveis séricos baixos, são seguros com relação aos efeitos adversos sistêmicos: seriam, portanto, uma alternativa eficaz aos AINEs orais?

Há um ceticismo na classe médica com relação à eficácia da aplicação tópica no alívio da dor. Os ensaios clínicos randomizados controlados de curta duração comparando AINEs tópicos com placebo, na sua maioria, demonstraram superioridade da droga. Naqueles com maior duração, observou-se uma tendência favorável aos AINEs tópicos. Porém, falhas metodológicas como o número de pacientes, a duração dos ensaios e interesses financeiros podem ter comprometido os resultados finais. A comparação entre os antiinflamatórios tópicos e orais ainda é limitada pelo pequeno número de ensaios. Há necessidade de mais estudos bem desenhados para afirmar que os AINEs tópicos são superiores ao placebo e tão efetivos quanto os orais. 


\section{REFERÊNCIAS}

1. Bagge E, Brooks P: Osteoarthritis in older patients: optimum treatment. Drugs Aging 7: 176-83, 1995.

2. Consenso Brasileiro para o tratamento da osteoartrite. Rev Bras Reumatol 42: 371-4, 2002.

3. American College of Rheumatology. Recommendations for the medical management of osteoarthritis of the hip and knee. 2000 Update. American College of Rheumatology Subcommittee on Osteoarthritis Guidelines. Arthritis Rheum 43: 1905-15, 2000.

4. Pendetlon A, Arden N, Dougados $M$ et al: EULAR recommendations for the management of knee osteoarthritis: report of a task force of the Standing Committee for International Clinical Studies Therapeutic Trials (ESCISIT). Ann Rheum Dis 59: 936-44, 2000.

5. Wynne HA, Campbell M: Pharmacoeconomics of non steroidal anti-inflammatory drugs (NSAIDS). Pharmaco Economics 3: 107-23, 1994.

6. Heyneman CA, Liday CL, Wall GC: Oral versus Topical NSAIDS in Rheumatic Diseases. Drugs 60: 555-74, 2000.

7. McNeill SC, Potts RO, Francouer ML: Local enhanced topical delivery (LETD) of drugs: does it truly exist? Pharm Res 9: 1422-7, 1992.

8. Mikulak SA, Vangsness CT, Nimni ME: Transdermal delivery and accumulation of indometacin in subcutaneous tissues in rats. J Pharmacol 50: 153-8, 1992.

9. Goodman LS, Gilman AG: As bases farmacológicas da terapêutica, 9 ${ }^{\underline{a}} \mathrm{Ed}$, Editora Mc Graw Hill do Brasil, 1996.

10. Squire I, Lees K: Topical drug delivery. Practioner 235: 203-6, 1992.

11. Algozzine GJ, Stein GH, Doering PL, Araújo OE, Akin KC: Trolamine salicylate in Osteoarthritis of the Knee. JAMA 247: 1311-3, 1982.

12. Shackel NA, O Day R, Kellet B, Brooks PM: Cooper-salicylate gel for pain relief in osteoarthritis: a randomized controlled trial. MJA 167: 134-6, 1997.

13. Dreiser RL, Tisne-Camus N: DHEP plasters as a topical treatment of knee osteoarthritis: A double blind placebo controlled study. Drugs Exp Res 19: 117-23, 1993.

14. Grace D, Rogers J, Skeith K, Anderson K: Topical Diclofenac versus Placebo: A double blind, Randomized Clinical Trial in Patients with osteoarthritis of the Knee. J Rheumatol 26: 265963, 1998.

15. Brühlmann P, Michel BA: Topical diclofenac patch in patients with osteoarthritis: A randomized, double-blind, controlled clinical trial. Clin Exp Rheumatol 21: 193-8, 2003.

16. Ottilinger B, Gömör B, Michel BA, Pavelka K, Beck W: Efficacy and safety of eltenac gel in the treatment of knee osteoarthritis. Osteoarthritis Cartilage 9: 272-80, 2001.

17. Lequesne MG: The algofunctional indices for hip and knee osteoarthritis. J Rheumatol 24: 779-81, 1997.

18. Rovensky J, Micekova D, Gubzoba Z et al: Treatment of knee osteoarthritis with topical non-steroidal anti-inflammatory drug. Results of randomized, double-blind, placebo-controlled study on the efficacy and safety of a 5\% ibuprofen cream. Drugs Exp Clin Res 27: 209-11, 2001.

19. Trnavsky K, Fischer M, Vogtle-Junkert U, Schreyger F: Efficacy and safety of $5 \%$ ibuprofen cream treatment in knee osteoarthritis. J Rheumatol 31: 565-72, 2004.

20. Bookman AAM, Williams KS, Shainhouse JZ: Effect of diclofenac solution for relieving symptoms for primary osteoarthritis of the knee. A randomized Controlled Trial. CMAJ 171: 333-8, 2004.

21. Roth SH, Shainhouse JZ: Efficacy and safety of a diclofenac solution (Pennsaid) in the Treatment of Primary Osteoarthritis of the knee. A Randomized, Double-Blind, Vehicle-Controlled Clinical trial. Arch Intern Med 164: 2017-23, 2004.

22. Baer PA, Thomas LM, Shainhouse JZ: Treatment of osteoarthritis of the knee with a topical diclofenac solution. A randomized controlled trial of 6-week trial solution. A randomized controlled trial of 6-week trial. BMC 6: 44, 2005.

23. Bellamy N, Kirwan J, Boers $M$ et al: Recommendations for a core set of outcome measures for future phase III clinicals trials in knee, hip and hand osteoarthritis: consensus development at OMERACT III. J Rheumatol 24: 799-802, 1997.

24. Bellamy N, Buchanan WW, Goldsmith CH, Campbell J, Sttit LW: Validation study of WOMAC: a health status instrument for measuring clinically-important patient relevant outcomes following anti-rheumatic drug therapy in patients with osteoarthritis of the hip or knee. J Rheumatol 15: 1833-40, 1988.

25. Moore RA, Tramèr MR, Wiffen PJ, McQuay HJ: Quantitive systematic review of topically applied non-steroidal antiinflammatory drugs. BMJ 316: 333-8, 1998.

26. Lin J, Zhang W, Jones A, Doherty M: Efficacy of topical non-steroidal anti-inflammatory drug in the treatment of osteoarthritis: meta-analysis of randomized controlled trials. BMJ 329: 324-47, 2004.

27. Tsuyama N, Kurokawa T, Hihei T: Clinical evaluation of L-141 topical agent on osteoarthrosis deformans of the knees. Clin Med 1: 697-729, 1985.

28. Sandelin J, Harilainen A, Crone H, Hamberg P, Forsskahl B, Tamelander G: Local NSAID Gel (Eltenac) in the Treatment of Osteoarthritis of the Knee. Scand J Rheumatol 26: 287-92, 1997.

29. Dickson DJ: A double-blind evaluation of topical piroxicam gel with oral ibuprofen in osteoarthritis of the knee. Curr Ther Res 49: 199-207, 1991.

30. Tugwell PS, Wells GA, Shainhouse JZ: Equivalence of a Topical Diclofenac Solution (Pennsaid) Compared with Oral Diclofenac in Syntomatic Treatment of Osteoarthritis of the Knee. A randomized Controlled Trial. J Rheumatol 31: 200212, 2004.

31. Mukherjee D, Nissen SE, Topol EJ: Risk of cardiovascular events associated with selective cox-2 inhibitors. JAMA 286 : 954-9, 2001. 